\title{
Parametric study of the conditions of supershear crack propagation in brittle materials
}

Cite as: AIP Conference Proceedings 1683, 020209 (2015); https://doi.org/10.1063/1.4932899

Published Online: 27 October 2015

E. V. Shilko, S. G. Psakhie, and V. L. Popov

ARTICLES YOU MAY BE INTERESTED IN

Structure of welded joints obtained by contact weld in nanostructured titanium

AIP Conference Proceedings 1683, 020083 (2015); https://doi.org/10.1063/1.4932773

Parametric study of a PULSCO vent silencer

The Journal of the Acoustical Society of America 136, 2305 (2014); https://

doi.org/10.1121/1.4900342

Formation of low-frequency harmonics on the surface of liquid hydrogen and helium in a turbulent regime

Low Temperature Physics 41, 163 (2015); https://doi.org/10.1063/1.4915913

\section{Lock-in Amplifiers up to $600 \mathrm{MHz}$}
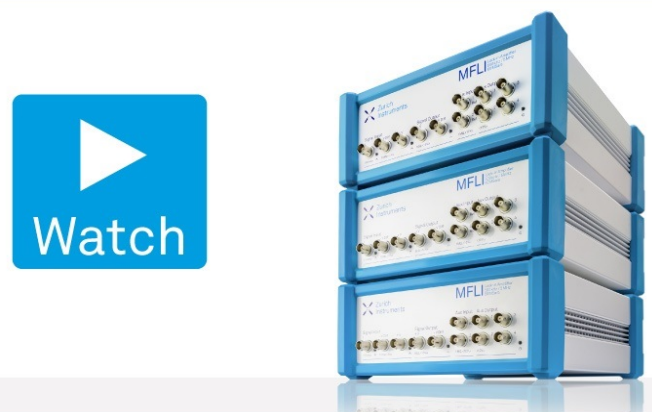


\title{
Parametric Study of the Conditions of Supershear Crack Propagation in Brittle Materials
}

\author{
E. V. Shilko ${ }^{1,2, a)}$, S. G. Psakhie ${ }^{1,2, b)}$, and V. L. Popov ${ }^{1,3, c)}$ \\ ${ }^{1}$ Institute of Strength Physics and Materials Science SB RAS, Tomsk, 634055 Russia \\ ${ }^{2}$ National Research Tomsk State University, Tomsk, 634050 Russia \\ ${ }^{3}$ Berlin University of Technology, Berlin, 10623 Germany

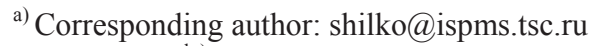 \\ b) sp@ispms.tsc.ru \\ c) v.popov@tu-berlin.de
}

\begin{abstract}
The paper is devoted to the numerical analysis of the conditions of acceleration of dynamically propagating longitudinal shear cracks from sub-Rayleigh to intersonic/supershear velocities. We showed that an ability of the initial crack to propagate in supershear regime can be predicted with use of the empirically derived dependence of the geometrical criterion of sub-Raleigh-to-intersonic transition on material and crack parameters.
\end{abstract}

\section{INTRODUCTION}

One of the topical issues of deformable solid and fracture mechanics is a question about physically admissible speed of dynamic crack growth. In conventional analytical models of fracture mechanics the crack growth rate cannot exceed the speed of the Rayleigh wave $V_{R}$ in the material. However, since the 1970s, a large number of laboratory and field data as well as the results of computer-aided simulations were published, which show that longitudinal shear cracks in brittle materials are able to propagate at velocities exceeding the speed of transverse elastic wave $V_{\mathrm{S}}$ and close to the speed of the longitudinal elastic wave $V_{\mathrm{P}}$. This dynamic regime of crack propagation is called intersonic or supershear.

In recent decades, the traditional "sub-Rayleigh" (at a velocity below $V_{\mathrm{R}}$ ) and the "supershear" regimes of dynamic (unstable) growth of longitudinal shear cracks in natural and technical systems were the subjects of a large number of experimental, theoretical and numerical studies [1-5]. Intensive treatment of this problem started from pioneering works of Burridge [1] and Andrews [2]. In particular, Andrews first discovered main features of longitudinal shear crack propagation with use of finite element model. He has shown that initially the dynamic rupture is propagating at a significant fraction of the Rayleigh wave speed. The stress peak in the crack tip arises during the initial phase of unstable crack growth. It propagates ahead of the rupture front at the shear wave velocity $V_{\mathrm{S}}$ (faster than $V_{\mathrm{R}}$ ) and gradually becomes higher. Under certain initial conditions the magnitude of the stress peak may reach yield strength and induce secondary rupture ahead the main crack at some distance from the crack tip [2, 3]. This secondary crack is capable to propagate faster than $V_{\mathrm{S}}$ (in supershear regime).

The phenomenon of stress peak induced acceleration of shear cracks towards the longitudinal wave speed is fundamental as it is observed numerically or instrumentally at various spatial scales from the atomic one [3] to the scale of tectonic faults $[4,5]$. Therefore, understanding of the conditions providing the ability of longitudinal shear crack to develop faster than the speed of transverse elastic wave $V_{\mathrm{S}}$ is the key to prediction of the regime of dynamic fracture of solids at different spatial scales under shear-dominated loading. Presented study is devoted to the numerical analysis of this problem. The particle-based movable cellular automaton (MCA) method [6] was used as a tool for investigation. 


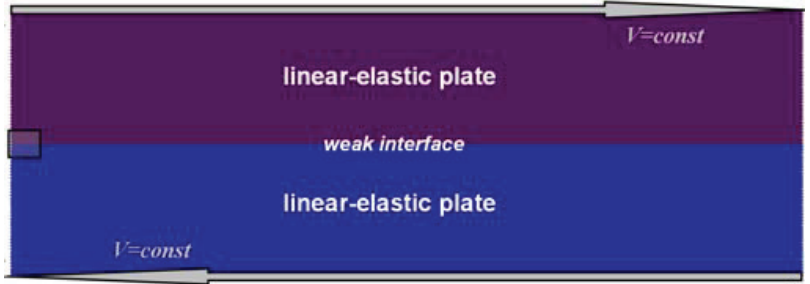

(a)

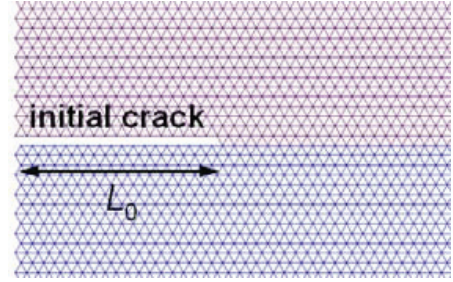

(b)

FIGURE 1. Structure and scheme of loading of the model sample (a); enlarged picture of small black rectangle in the left part of (a) containing initial interface crack (b)

\section{PROBLEM STATEMENT}

The numerical study was carried out with use of the two-dimensional model slab (Fig.1a), which consists of two bonded parts. Parts have the same properties and are isotropic, linear-elastic and high-strength. Ideal bonding between the parts was assumed. Interface strength was taken to be much smaller than the strength of material of the plates.

A short initial section (preliminary crack) at the interface was cut by means of breaking bonds between corresponding linked elements belonging to different parts of the slab (Fig. 1b).

To simulate shear loading, the upper and the lower boundaries of the slab were displaced in opposite directions along the interface line with low constant velocity $V_{\text {load }}$ (Fig. 1a). This loading scheme models the condition of simple shear of the system in quasi-static regime. Periodic boundary conditions were applied to opposite vertical (side) faces to avoid the influence of contortions at these faces of the slab. The plane strain condition was applied to the described model.

Under such a loading the course of deformation of the slab consists of two stages. In the first stage the slab is elastically deformed and accumulates elastic energy. Maximum shear stresses in the specimen are concentrated at the crack tip. Upon reaching the threshold value of shear stress $\tau_{0}$ (shear strength $\tau_{0}$, which depends on the length of the initial crack and amounts a portion of the shear strength of intact interface) the second stage related to the dynamic crack propagation at the interface begins.

\section{SIMULATION RESULTS AND DISCUSSION}

In the previous study the authors have discovered the mechanism of acceleration of dynamically propagating shear cracks in brittle materials towards the longitudinal wave speed $V_{\mathrm{P}}$ [7]. It has been shown that in the initial phase of dynamic crack growth the collective vortex-like elastic motion of material particles (hereinafter referred to as elastic vortex) is formed ahead of the tip of growing crack. After the formation the elastic vortex becomes a selfdependent scale-invariable dynamic object that travels at the speed of elastic shear wave (faster than the crack tip). Therefore during the course of crack and vortex propagation the elastic vortex moves away from the crack tip and may separate from it. An important feature of the elastic vortex is a concentration of shear stress, which gradually increases until the moment of vortex separation from the crack. This feature determines the ability of shear cracks to accelerate to velocities close to $V_{\mathrm{P}}$. Such acceleration takes place if the magnitude of elastic stresses in elastic vortex reaches the magnitude of the material shear strength before the moment of vortex separation from the crack [7].

The present study is a development of the investigation described above. It was focused on the detail analysis of the conditions of crack acceleration towards the longitudinal wave speed. The study has shown that the maximum achieved magnitude of the stress peak in elastic vortex is proportional to the shear strength $\tau_{0}$ of the slab with initial crack, or, what is the same, to the root of accumulated elastic strain energy $E_{0}\left(\tau_{0}=\sqrt{2 E_{0} G}\right.$, where $G$ is the shear modulus of material). Parameters $E_{0}$ and $\tau_{0}$ are directly connected to geometrical characteristics of initial crack (particularly, $E_{0}$ is inversely proportional to its length). Due to scale invariance of crack and vortex propagation the parameters $E_{0}$ and $\tau_{0}$ (and hence the maximum magnitude of the stress peak) are the functions not of individual dimensional geometrical characteristics of initial crack but of dimensionless parameter connecting dimensional geometrical characteristics. Analysis of the simulation results revealed a dimensionless geometrical criterion of crack transition to supershear regime of dynamic propagation for the case of straight cracks. It is a ratio of the initial 
(preliminary) crack length $L_{0}$ to effective crack thickness $D: P=L_{0} / D$. Longitudinal shear crack is able to propagate in supershear regime only if the value of the parameter $P$ does not exceed a certain critical (threshold) value $P_{\text {cr }}$. Note that the existence of a dimensionless parameter $P$, which determines the influence of the geometrical characteristics of a crack on $\tau_{0}$ (and, consequently, on the equivalent stress peak), confirms the scale invariant nature of dynamic growth of longitudinal shear cracks [7].

The threshold value of geometrical parameter $P$ is a function of the material parameters. These parameters for the elastic-brittle materials are elastic constants, density, dynamic viscosity and strength of the interface. The special research was carried out to define the functional form of the dependence of the critical value $\left(P_{\mathrm{cr}}\right)$ of a dimensionless geometric parameter $P$ on magnitudes of these material characteristics. The simulation results showed that in brittle materials the value of $P_{\mathrm{cr}}$ is determined mainly by elastic constants of the material and its density. Both the dynamics of unstable crack propagation and stress concentration growth in the elastic vortex ahead of the crack tip are controlled by elastic energy fluxes. The rate of energy flow is determined by elastic wave speeds (elastic wave speeds are material parameters which depend on the ratios of elastic moduli to material density). Therefore from physical point of view stress concentration in the elastic vortex is determined by the following differences between speeds of different elastic waves:

(i) The difference between shear wave speed $V_{\mathrm{S}}$ and Rayleigh wave speed $V_{\mathrm{R}}$ (which is an upper limit of dynamic crack propagation in sub-Rayleigh regime). The difference $\left(V_{\mathrm{S}}-V_{\mathrm{R}}\right)$ characterizes time interval between the moments of elastic vortex nucleation and separation from the crack. The longer time interval the higher stress concentration in the vortex and vice versa (the larger speed difference the smaller stress concentration in the vortex).

(ii) Difference between longitudinal wave speed $V_{\mathrm{P}}$ and shear wave speed $V_{\mathrm{S}}$. During the course of elastic vortex propagation a fraction of its elastic strain energy outflows in surrounding areas by longitudinal elastic waves. So, this difference characterizes energy loss by elastic vortex.

From this point of view the critical value of the dimensionless geometrical crack parameter (or what is the same the critical value of shear strength) should depend on the sum of these two differences, namely on the difference between longitudinal and Rayleigh wave speeds $\left(V_{\mathrm{P}}-V_{\mathrm{R}}\right)$.

Figure 2a shows examples of numerically determined dependences of dimensionless geometrical parameter $P$ on elastic wave difference. Here each set of points connected by line was obtained by varying the density and Young modulus of the slab material while maintaining constant value of Poisson ratio. Different sets correspond to different Poisson ratios. All the sets corresponding to different Poisson ratios are parallel to each over (they are shifted along the vertical axis). The offset is proportional to the Poisson ratio.

These curves can be merged into one so-called "master curve" when using derived geometrical parameter instead of original one (Fig. 2b). Total set of points corresponding to different values of elastic constants and densities is approximated well by the following empirical equation, which is inverse proportion to elastic wave difference:

$$
P_{\mathrm{cr}}=\frac{A}{1+B\left(V_{\mathrm{P}}-V_{\mathrm{R}}\right)}+3(5 v-1)
$$

where $A$ and $B$ are constants, $v$ is a Poisson ratio. Expression (1) determines critical value of dimensionless geometrical crack parameter (and hence the critical density of accumulated elastic strain energy) via elastic wave velocity difference and Poisson's ratio.

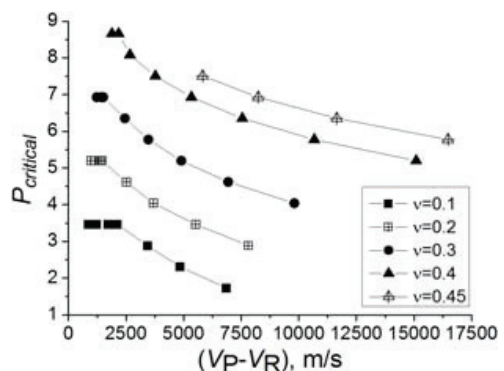

(a)

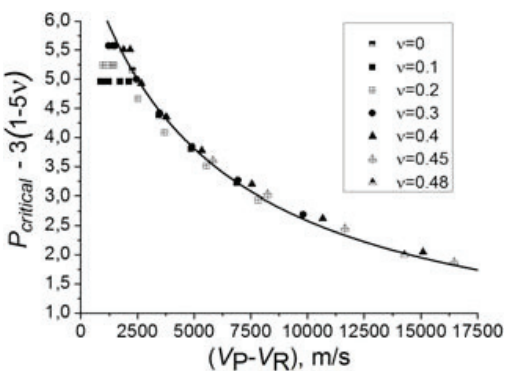

(b)

FIGURE 2. Dependences of the value of critical geometrical parameter $P_{\mathrm{cr}}$ on elastic wave difference for slab materials characterized by different ratios $E / \rho$ and Poisson ratios $v$ (a) and the "master curve" (b) 
The relation is valid in the very wide ranges of elastic constants and densities except the region of very small values of velocity difference. This region corresponds to extremely heavy or soft material. Here the dependence $P_{\mathrm{cr}}\left(V_{\mathrm{P}}-V_{\mathrm{R}}\right)$ tends to saturation.

The influence of other parameters of elastic-brittle material on the threshold value of geometrical parameter $P$ is much weaker. In particular, dependence of $P_{\mathrm{cr}}$ on dynamic viscosity $\mu$ has logarithmic form: $P_{\mathrm{cr}}=a-b \ln \mu$, where $a$ is determined by the expression (1) with constants $A$ and $B$ corresponding to the materials with zero viscosity. Note that hundred times increase in material viscosity leads to two times smaller change in the value of $P_{\mathrm{cr}}$ than variation of Poisson ratio from 0 to 0.5 .

At the same time the interface strength (which characterizes the density of elastic strain energy accumulated to the moment of crack propagation beginning) has no significant influence on $P_{\mathrm{cr}}$. In the carried out study the twoparametric Drucker-Prager fracture criterion was used. Special analysis has shown that absolute values of the parameters of this criterion and the ratio of these parameters determine crack velocity in sub-Rayleigh and supershear regimes but not the geometrical threshold of sub-Rayleigh-to-supershear transition.

So, the main material parameters governing the condition (threshold) of crack propagation in intersonic regime in brittle materials are difference between longitudinal and Rayleigh wave speeds and Poisson's ratio. Note that in elastic-plastic materials parameters of inelastic response determining intensity of localized energy dissipation are critically important.

\section{CONCLUSION}

The paper nicely complements numerous numerical and laboratory studies of dynamic mode II fracture as it shows that the condition of longitudinal shear crack acceleration towards longitudinal wave speed can be expressed in terms of a dimensionless geometrical parameter characterizing the initial crack. Moreover the importance of this result is related to its predictive ability. Numerically derived dependence (1) makes it possible to estimate critical value of dimensionless geometrical crack parameter for the considered brittle material. This allows one to forecast an ability of pre-existing crack (characterized by geometrical parameter $P$ ) in the considered material to propagate in supershear regime under shear-dominated loading.

Note that elastic vortex related mechanism of stress redistribution in solids determines not only the regularities of dynamic crack growth (including sub-Rayleigh-to-supershear transition [7]) but peculiarities of deformation of materials under dynamic loading as well [8]. For example recent results of numerical modeling demonstrate that the vortex related mechanism can be responsible for grain boundary migration and "hot spots" formation under highrate shear loading $[9,10]$ as well as for the formation of mechanically mixed layers in tribological systems [11].

\section{ACKNOWLEDGMENTS}

E.V. Shilko and V.L. Popov acknowledge financial support from the Russian Science Foundation grant 14-1900718 (Russia).

\section{REFERENCES}

1. R. Burridge, Geophys. J. R. Astr. Soc. 35, 439-455 (1973).

2. D. J. Andrews, J. Geophys. Res. 81, 5679-5687 (1976).

3. F. F. Abraham and H. Gao, Phys. Rev. Letters 84, 3113-3116 (2000).

4. X. Lu, N. Lapusta, and A. J. Rosakis, Geophys. J. Int. 177, 717-732 (2009).

5. M. Mello, H. S. Bhat, A. J. Rosakis, and H. Kanamori, Tectonophysics 493, 297-326 (2010).

6. S. G. Psakhie, E. V. Shilko, A. S. Grigoriev, et al., Eng. Fract. Mech. 130, 96-115 (2014).

7. S. G. Psakhie, E. V. Shilko, M. V. Popov, and V. L. Popov, Phys. Rev. E 91, 063302 (2015).

8. S. G. Psakhie, K. P. Zolnikov, A. I. Dmitriev, A. Yu. Smolin, and E. V. Shilko, Phys. Mesomech. 17(1), 15-22 (2014).

9. S. G. Psakh'e and K. P. Zol'nikov, Combust. Explo. Shock 34, 366-368 (1998).

10. P. R. Guduru, G. Ravichandran, and A. J. Rosakis, Phys. Rev. E 64, 036128 (2001).

11. A. I. Dmitriev and W. Osterle, Tribol. Int. 43, 719-727 (2010). 